\title{
ENUMERATIONS FOR PERMUTATIONS IN DIFFERENCE FORM
}

\author{
JOHN RIORDAN
}

1. Introduction. If $\left(p_{1}, p_{2}, \cdots, p_{n}\right)$ is a permutation of elements 1 to $n$, then $\left(\pi_{1}, \pi_{2}, \cdots, \pi_{n}\right)$ with $\pi_{j} \equiv p_{j}-j(\bmod n)$ is the corresponding difference form. Since $p_{1}+\cdots+p_{n}=1+2+\cdots+n$, it follows that $\pi_{1}+\pi_{2}+\cdots+\pi_{n} \equiv 0(\bmod n)$; hence the difference forms apart from order are partitions of $k n, k=0,1, \cdots, n-1$ with largest part $n-1$ and at most $n$ parts. Marshall Hall [1] has shown that every such partition corresponds to at least one permutation. Here it is shown that the number of these partitions is given by

$$
P_{0, n}=\frac{1}{n} \sum_{d \mid n} \phi(n / d)\left(\begin{array}{c}
2 d-1 \\
d
\end{array}\right)
$$

with summation over all divisors on $n$ (including 1 and $n$ ) and $\phi(n)$ the Euler totient function.

2. A partition enumerator. It is convenient to determine the enumerator for partitions with largest part $i$ and at most $n$ parts by use of a theorem of Pólya, as in [4]. Thus they are regarded as unordered arrangements on a line of elements each of which may have any of the values $0,1, \cdots, i$ (corresponding to a store enumerator $\left.1+x+\cdots+x^{i}\right)$ and with order equivalences for all operations of the symmetric group on $n$ elements. Then, if $P_{n}(x, i)$ is the enumerator, by the theorem

$$
P_{n}(x, i)=S_{n}\left(s_{1}, s_{2}, \cdots, s_{n}\right), \quad s_{k}=1+x^{k}+\cdots+x^{i k},
$$

with $S_{n}\left(x_{1}, x_{2}, \cdots, x_{n}\right)$ the cycle index of the symmetric group, which for present purposes may be taken as defined by$$
\sum_{n=0} S_{n}\left(x_{1}, x_{2}, \cdots x_{n}\right) y^{n}=\exp \left(x_{1} y+x_{2} \frac{y^{2}}{2}+\cdots+x_{n} \frac{y^{n}}{n}+\cdots\right) \text {. }
$$

Writing

$$
P(x, y)=\sum_{n=0} P_{n}(x, i) y^{n}
$$

and using (2) and (3), it is found that

$$
P(x, y)=1 /(1-y)(1-x y) \cdots\left(1-x^{i} y\right)
$$

a result which is immediate otherwise. Since

Received by the editors February 10, 1961. 


$$
(1-y) P(x, y)=\left(1-x^{i+1} y\right) P(x, x y)
$$

it follows from (4) that

$$
P_{n}(x, i)=\frac{1-x^{i+1}}{1-x} \frac{1-x^{i+2}}{1-x^{2}} \cdots \frac{1-x^{i+n}}{1-x^{n}}
$$

a result given by $\mathrm{P}$. A. MacMahon $[2$, p. 5$]$, who has also noticed $[2$, p. 66] the equivalent result, equation (2). By (2a)

$$
P_{n}(x, n-1)=P_{n-1}(x, n) ;
$$

by (2), this corresponds to the interesting identity

$$
S_{n}\left(s_{1, n-1}, \cdots, s_{n, n-1}\right)=S_{n-1}\left(s_{1, n}, \cdots, s_{n-1, n}\right)
$$

with $s_{k, i}=1+x^{k}+\cdots+x^{i k}$. Notice also that from (2a), on evaluating the indeterminate form,

$$
P_{n}(1, n-1)=\left(\begin{array}{c}
2 n-1 \\
n
\end{array}\right)
$$

Finally it may be noticed that the enumerator for compositions is obtained from the theorem as

$$
C_{n}(x, i)=\left(1+x+\cdots+x^{i}\right)^{n}
$$

since the group of equivalences consists solely of the identity (cycle index $\left.x_{1}^{n}\right)$.

3. Multisection of enumerators. The enumerator $P_{n}(x, n-1)$ gives as coefficient of $x^{m}, m=0,1, \cdots, n(n-1)$, the number of partitions of $m$ into at most $n$ parts and with largest part $n-1$. The partitions corresponding to permutations in difference form are for only those values of $m$ which are zero or multiples of $n$. To pick out such terms requires what DeMorgan [3] calls multisection of the series of terms in the enumerator, which is accomplished by simple properties of the roots of unity. Briefly if

$$
a(x)=a_{0}+a_{1} x+\cdots
$$

and $\alpha$ is a primitive $n$th root of unity, then the $i$ th $n$-sectional series

$$
a_{i, n}(x)=a_{i} x^{i}+a_{i+n} x^{i+n}+\cdots
$$

is given by

$$
a_{i, n}(x)=n^{-1} \sum_{j=1}^{n} \alpha^{-i j} a\left(\alpha^{i} x\right)
$$


Applied to the partition enumerator $P_{n}(x, n-1),(8)$ gives

$$
P_{i, n}(x, n-1)=n^{-1} \sum_{j=1}^{n} \alpha^{-i j} P_{n}\left(\alpha^{j} x, n-1\right)
$$

and in particular

$$
P_{i, n} \equiv P_{i, n}(1, n-1)=n^{-1} \sum_{j=1}^{n} \alpha^{-i j} P_{n}\left(\alpha^{i}, n-1\right)
$$

is the sum of the numbers of partitions of all integers congruent to $i$, modulo $n$.

Equation (9) seems not to have much to offer, but equation (10) does. First it is clear that the powers of $\alpha$ may be classified according to their period; there are $\phi(d)$ powers of period $d$, and, if $\beta_{1}, \beta_{2}$ are roots, each of period $d, P_{n}\left(\beta_{1}, n-1\right)=P_{n}\left(\beta_{2}, n-1\right)$. If $\beta^{d}=1$ and $d e=n$, then

$$
\begin{aligned}
s_{k}(\beta) & =1+\beta^{k}+\cdots+\beta^{k(d \sigma-1)} \\
& =\left(1+\beta^{k}+\cdots+\beta^{k(d-1)}\right)\left(1+\beta^{k d}+\cdots+\beta^{k d(\sigma-1)}\right)
\end{aligned}
$$

and since $1+\beta+\cdots+\beta^{d-1}=0$,

$$
\begin{aligned}
s_{k}(\beta) & =0, & & d \nmid k, \\
& =n, & & d \mid k .
\end{aligned}
$$

Hence, by (2)

$$
P_{n}(\beta, n-1)=S_{n}(0, \cdots, n, 0, \cdots n, \cdots), \quad \beta^{d}=1,
$$

the nonzero entries in $S_{n}$ occurring at positions $j d, j=1,2, \cdots$.

If in (3) $x_{k}=0, d \nmid k, x_{k}=x, d \mid k$, then

$$
\begin{aligned}
\sum_{n=0} S_{n}\left(x_{1}, \cdots, x_{n}\right) y^{n} & =\exp (x / d)\left(y^{d}+\frac{y^{2 d}}{2}+\cdots\right) \\
& =\left(1-y^{d}\right)^{-x / d} \\
& =\sum_{j=0}\left(\begin{array}{c}
j-1+x d^{-1} \\
j
\end{array}\right) y^{j d} .
\end{aligned}
$$

Hence

$$
P_{n}(\beta, n-1)=\left(\begin{array}{c}
2 e-1 \\
e
\end{array}\right), \quad \beta^{d}=1, \quad d e=n,
$$

and by (10) with $i=0$, 


$$
P_{0, n}=n^{-1} \sum_{d \mid n} \phi(d)\left(\begin{array}{c}
2 e-1 \\
e
\end{array}\right), \quad d e=n,
$$

the result stated in the introduction.

The $P_{i, n}$ may all be expressed in terms of the $P_{0, n}$. Thus for $n=p$, a prime,

$$
P_{i, p}=P_{0, p}-1, \quad i=1,2, \cdots, p-1 .
$$

For $n=p q, p$ and $q$ prime,

$$
\begin{array}{lrl}
P_{i, p q}=P_{0, p q}-P_{0, p}-P_{0, q}+1, & i \nmid p, q, \\
P_{j p, p q}=P_{0, p q}-P_{0, p}, & j=1,2, \cdots, q-1, \\
P_{j q, p q}=P_{0, p q}-P_{0, q}, & j=1,2, \cdots, p-1 .
\end{array}
$$

For $n=p^{k}$,

$$
P_{p^{i}, p^{k}}=P_{0, p^{k}}-P_{0, p^{k-j+1}}, \quad j<k .
$$

Finally it may be noticed that the corresponding composition sums $C_{i, n}$ (defined as in (10)) all have the common value

$$
C_{i, n}=n^{n-1},
$$

since $C_{n}\left(\alpha^{j}, n-1\right)=0, j<n$ and $C_{n}(1, n-1)=n^{n}$. Hence they are equinumerous with fully point-labeled rooted trees.

\section{REFERENCES}

1. Marshall Hall, Jr., A combinatorial problem on Abelian groups, Proc. Amer. Math. Soc. 3 (1952), 548-587.

2. P. A. MacMahon, Combinatory analysis, Vol. II, Cambridge Univ. Press, Cambridge, 1916.

3. Augustus DeMorgan, The differential and integral calculus, Robert Baldwin, London, 1942.

4. John Riordan, The combinatorial significance of a theorem of Polya, J. Soc. Indust. Appl. Math. 5 (1957), 225-237.

Bell Telephone laboratories, Inc. 